\title{
Developmental shifts in computations used to detect environmental controllability
}

\author{
Hillary A. Raab ${ }^{1}$, Careen Foord ${ }^{1}$, Romain Ligneul ${ }^{2^{*}}$, Catherine A. Hartley ${ }^{1,3^{*}}$ \\ ${ }^{1}$ Department of Psychology, New York University, New York, NY \\ ${ }^{2}$ Champalimaud Research, Champalimaud Center for the Unknown, Lisbon, Portugal \\ ${ }^{3}$ Center for Neural Science, New York University, New York, NY \\ ${ }^{*}$ Equal author contribution
}

Correspondence concerning this manuscript should be addressed to Catherine A. Hartley (cate@nyu.edu), Department of Psychology, New York University, 6 Washington PI., New York, NY 10003.

Keywords: Causal inference, Environmental controllability, Learning, Cognitive development 


\section{ABSTRACT}

Accurate assessment of environmental controllability enables individuals to adaptively adjust their behavior - exploiting rewards when desirable outcomes are contingent upon their actions and minimizing costly deliberation when their actions are inconsequential. However, it remains unclear how estimation of environmental controllability changes from childhood to adulthood. Ninety participants (ages 8-25) completed a task that covertly alternated between controllable and uncontrollable conditions, requiring them to explore different actions to discover the current degree of environmental controllability. We found that while children were able to distinguish controllable and uncontrollable conditions, accuracy of controllability assessments improved with age. Computational modeling revealed that whereas younger participants' controllability assessments relied on evidence gleaned through random exploration, older participants more effectively recruited their task structure knowledge to make highly informative interventions. Age-related improvements in working memory mediated this qualitative shift toward increased use of an inferential strategy. Collectively, these findings reveal an age-related shift in the cognitive processes engaged to assess environmental controllability. Improved detection of environmental controllability may foster increasingly adaptive behavior over development by revealing when actions can be leveraged for one's benefit.

\section{INTRODUCTION}

Over the course of our lives, we are faced with the challenge of determining when our actions are consequential. In environments that are highly controllable, our actions can reliably produce a particular outcome, whereas in uncontrollable environments, our actions have no causal influence. By estimating the degree of contingency between actions and their resulting outcomes, individuals can assess the extent of control they have over their environment, and adapt their behavior accordingly (Boureau et al., 2015; Dorfman \& Gershman, 2019; Frankenhuis et al., 2013; Haggard, 2017; Huys \& Dayan, 2009; Moscarello \& Hartley, 2017). For example, imagine a child whose parents typically reward her good behavior at the dinner table with dessert. This child might be on her best behavior when eating with her parents because she has learned that her actions directly influence the likelihood of her getting a treat. When at her friend's house, this same girl might assume that, just like at home, her behavior at the table will determine whether she can have dessert. Thus, she might expend energy minding her manners even if, in actuality, meals at her friend's home always finish with dessert. Rather than simply generalizing prior beliefs about the controllability of the environment, individuals can make informative interventions to reveal the actual degree of contingency between actions and outcomes (Lagnado \& Sloman, 2004; Pearl, 2009; Sloman \& Lagnado, 2005; Steyvers et al., 2003). Returning to the girl in the example, always being on her best behavior when at her friend's house cannot test her assumption that her table manners are consequential. To disambiguate whether good behavior, or simply an indulgent parent, is responsible for her getting dessert, a more informative intervention would be for her to occasionally behave poorly. By varying her behavior - that is, by exploring - she can obtain better evidence for assessing whether her actions influence the outcomes she receives when the true causal structure of the environment is unknown. 
Experiences of environmental control have a profound effect on learning and behavior, shaping developmental trajectories from an early age (Frankenhuis et al., 2013; Gunnar, 1980; Mineka \& Hendersen, 1985). Infants as young as two months old are sensitive to when outcomes are contingent upon their own actions (Nadel et al., 1999), and early contingent social interaction influences diverse aspects of social and cognitive development, including language learning (Goldstein et al., 2003; Takahashi et al., 2017) and caregiver attachment (Mesman et al., 2009). Perceptions of control, whether actual or illusory, are often experienced as subjectively rewarding, a phenomenon proposed to underpin an intrinsic motivation for controllability (Leotti et al., 2010; Ly et al., 2019). Motivation to exert control is proposed to be a key driver of development (Heckhausen, 2011; Kelso, 2016). Control facilitates learning and memory (Chambon et al., 2020; Cockburn et al., 2014), even from a young age (Katzman \& Hartley, 2020; Ruggeri et al., 2019), and artificial agents equipped with a drive to exert control develop more complex action repertoires (Klyubin et al., 2005; Salge et al., 2014). During adolescence, greater parental independence provides increased opportunity to make autonomous decisions, which may make accurate recognition of the contexts in which one's actions are most consequential particularly beneficial (Hansen \& Jessop, 2017). Collectively, these findings suggest that detection of environmental controllability provides foundational knowledge about the structure of the environment that supports the development of an individual's behavioral repertoire.

An extensive literature has investigated the development of causal inference more broadly (Gopnik et al., 2004; Sobel \& Legare, 2014). Preschoolers, and even infants as early as 8 weeks of age, can perform actions to reveal the causal structure of their environments (Cook et al., 2011; Sobel \& Sommerville, 2010; Watson \& Ramey, 1972). However, younger children often choose less informative interventions in more complex tasks (Cook et al., 2011; Kuhn et al., 1995; Rieber, 1969). The ability to identify and select informative interventions to reveal the causal structure of the environment continues to develop across childhood and into adolescence (Nussenbaum et al., 2020; Rieber, 1969). While making causal interventions to test hypotheses about action-outcome contingencies is an efficient way to learn, it may require the use of a mental model of the environment, an ability that undergoes continued refinement from childhood into young adulthood (Decker et al., 2016; Potter et al., 2017; Raab \& Hartley, 2018). Working memory, a cognitive process critical for maintaining and manipulating these mental representations of environmental structure (Otto et al., 2013), shows similar age-related improvements across adolescence (Amso et al., 2014; Crone et al., 2006) and may underpin both the ability to make informative interventions and to use the resulting evidence to infer causal structures. Thus, developmental improvements in the cognitive processes that support effective intervention strategies and causal inference suggest that accurate assessment of the degree of environmental controllability may also exhibit marked age-related changes from childhood to adulthood. However, given that the ability to control the environment is typically a premise in causal learning studies, it remains unclear how the ability to test the hypothesis of whether or not one's own actions actually have causal efficacy might change with age. 
As children may not be as adept as adults at making informative causal interventions, they may instead rely on other cognitive processes that can effectively support the detection of environmental controllability from an early age. Learning of statistical regularities present in the environment emerges early in development (Amso \& Davidow, 2012; Meulemans et al., 1998; Saffran et al., 1996). Younger individuals also tend to exhibit increased stochasticity in action selection (Gopnik et al., 2015; Lucas et al., 2014; Meder et al., 2020; Nussenbaum \& Hartley, 2019; Sumner et al., 2019). In lieu of making highly informative interventions, younger individuals may discern the contingency structure of the environment by applying their robust statistical learning abilities to the observations of actions and outcomes generated through random exploration (Kushnir \& Gopnik, 2005; Sobel \& Kushnir, 2006). As children inherently have less experience than adults across many situations, the coupling of greater intrinsic behavioral variability with robust statistical learning ability may be particularly advantageous, as it provides a generalizable strategy for detecting control across diverse environments (Gopnik et al., 2015).

An environment can be considered controllable to the extent that actions bring about specific state transitions. Such a causal coupling between the actions of an agent and the states of the environment can be estimated by comparing the predictability of upcoming states (e.g., dessert) when only considering previous states (e.g., dinner) versus when considering both previous states and actions (e.g., dinner at which one showed good manners) (Barnett et al., 2009; Ligneul, 2021). Incorporating actions into the predictive process will only improve one's forecasts in controllable environments, where actions causally influence state transitions. Thus, computational models that compare the accuracy of state predictions based on states alone, versus those based on states and actions, can provide a formal account of the controllability estimation process (Dorfman \& Gershman, 2019; Ligneul et al., 2020). Importantly, predictions about upcoming states can be made in different ways depending on the task and the amount of knowledge one has about its underlying structure (Dorfman \& Gershman, 2019; Huys \& Dayan, 2009; Ligneul et al., 2020). By comparing how distinct computational models fit participants' behavior at different stages of development, we can characterize the nature of the learning processes through which participants detect environmental controllability.

Here, we asked whether children, adolescents, and adults differed in their ability to detect changes in environmental controllability and to use informative interventions to reveal these shifts in causal structure. Ninety individuals, aged 8-25, performed a child-friendly adaptation of the 'Explore-and-Predict' task - a novel task designed to assess individuals' ability to estimate the degree of environmental controllability in a dynamic, yet predictable, context and how these beliefs are shaped by the informativeness of interventions (Ligneul et al., 2020). Throughout the task, participants flew with one of two pilots on different colored planes around a set of islands. A key feature of the task was that participants' choices determined where one of the pilots would fly (i.e., the controllable condition), but critically had no influence on the flight path of the other pilot (i.e., the uncontrollable condition). The two pilot conditions alternated covertly throughout the task, and participants were instructed that they could earn more points if they accurately tracked the current condition. On exploratory trials, participants could make active interventions to infer whether their actions were consequential. On prediction trials, participants 
were asked to report the likely subsequent state, revealing these controllability beliefs. We used computational modeling to assess both the complexity of participants' task structure representations and the degree to which their estimation process reflected use of an inferential strategy. We hypothesized that the detection of environmental controllability would improve with age, reflecting a qualitative shift in the cognitive processes involved in controllability estimation across development. Whereas adults' greater ability to maintain complex task structure representations in working memory may facilitate the use of informative interventions to infer controllability, younger individuals may, instead, rely on random exploration to generate evidence that can reveal the contingency structure of the environment.

\section{METHODS}

\section{Participants}

As we did not know the size of our hypothesized effect, we targeted a sample size of 90 participants, based on previous developmental studies using computational modeling of choice behavior to characterize age-related cognitive changes (Cohen et al., 2020; Decker et al., 2015; Nussenbaum et al., 2020; Palminteri et al., 2016). Ninety-three participants, recruited from the New York City metropolitan area, completed questionnaires and a computer-based learning task. We excluded three participants due to technical errors during the task. Our final sample of 90 participants included thirty children (8-12 years old, mean $=10.46$, s.d. $=1.55$, female $n=$ $15)$, thirty adolescents (13-17 years old, mean age $=15.44$, s.d. $=1.44$, female $n=15)$, and thirty adults $(18-25$ years old, mean age $=22.06$, s.d. $=2.30$, female $n=15)$. The breakdown of participants' self-identified race was as follows: $35.56 \%$ Caucasian/White, $16.67 \%$ African American/Black, 25.56\% Asian, and 22.22\% Mixed Race. $13.33 \%$ reported identifying as Hispanic/Latinx. Participants' total combined family incomes for the previous twelve months ranged from less than $\$ 5,000$ to $\$ 100,000$ or greater. Exclusionary criteria for the study included colorblindness, a diagnosis of psychiatric or learning disorders or disabilities, or the current use of psychoactive medications. All participants had normal or corrected-to-normal vision. This study was approved by the New York University Committee on Activities Involving Human Subjects. All participants or a parent, in the case of a minor, provided written consent prior to participation. Participants were compensated \$15/hour and were instructed that they would receive a bonus payment based on their performance. In reality, all participants earned a $\$ 5$ bonus regardless of their performance.

\section{Assessment of Control Task}

We assessed participants' ability to detect the causal structure of their environment using a task that covertly switched between a controllable and uncontrollable condition. This task was adapted from a previous version of the paradigm created for adults (Ligneul et al., 2020). To make the study suitable for children, we decreased the complexity of the task by removing a second set of controllable and uncontrollable task transitions and included a child-friendly narrative. Participants acted as a travel guide for other passengers by providing them information about flights to three destinations (i.e., the volcano island; the palm tree island; the lighthouse island). There were two pilots, representing the controllable or uncontrollable condition, who could fly passengers around on three different colored planes (i.e., pink, green, 
orange). One of the pilots flew to the destination based on a specific route, whereas the other pilot flew to the destination depending on the color of the plane chosen (Fig. 1a). Participants were never told which pilot was flying the plane, encouraging them to discover the current condition through their choices on exploratory trials. The participant's goal was to learn where the plane would fly and which pilot was currently flying, in order to help other passengers find their way (Fig. 1b). To provide a general incentive to perform well throughout the task, participants were told that they would earn treasure for helping other passengers successfully reach their destinations, and that the more treasure they earned, the more bonus money they would receive. To avoid any potential age differences in the subjective value of the bonus, participants were not told the amount of bonus money that they could possibly earn or the conversion rate of treasure into money. The computerized task was coded in Psychtoolbox v3.0.14 with Matlab v2016b.

\section{Exploratory Trials}

On each exploratory trial, participants saw the current island and two planes (Fig. 1c). Participants chose one of the planes to see where it would fly. Notably, each pilot flew according to a particular rule. In the controllable condition, the color of the plane that the participant selected determined the destination. Thus, participants' choices determined where the "color" pilot would fly (e.g., selecting the pink plane led to the palm tree, whereas selecting the green plane led to the volcano). In the uncontrollable condition, the pilot flew around the islands according to a particular route. The "route" pilot flew to the next destination in the route irrespective of the color plane that the participant had selected (e.g., volcano to the lighthouse to the palm tree). Prior to playing the game, participants were explicitly instructed about the flight routes that governed each pilot's flying patterns. As the pilots alternated covertly, participants could use the knowledge about the transition structure for each pilot to discover the current condition. Critically, only one of the two planes displayed on each exploratory trial could be diagnostic as to the current condition. Choosing the other plane was not informative, as it flew to the same island in both the controllable and uncontrollable conditions. In the example shown in Fig. 1c, selecting the pink plane would lead to the palm tree in the controllable condition and in the uncontrollable condition. Thus, from that departure island, selecting the pink plane contributes to learning transition probabilities but is uninformative as to the current condition. Instead, the green plane is the informative choice (Fig. 1d). In the uncontrollable condition, selecting the green plane would lead to the palm tree, because the "route" pilot visits the palm tree after the lighthouse. However, in the controllable condition, the green plane would lead to the island with the volcano. Only by selecting this diagnostic choice (i.e., the green plane) could participants obtain information that enables discrimination between conditions. 
a

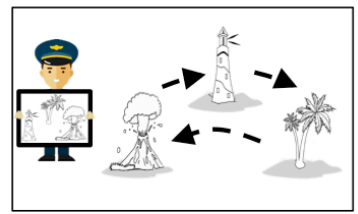

Uncontrollable

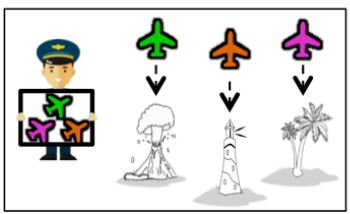

Controllable b



C

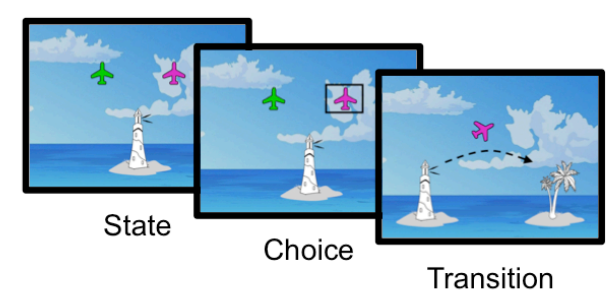

d

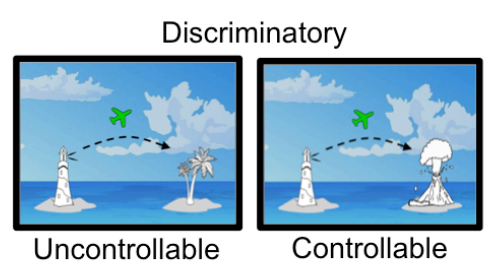

Exploratory Choice Types

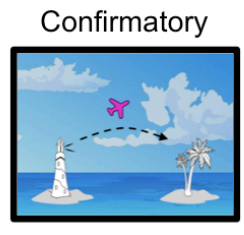

Figure 1. Task Design. (a) Two pilots fly participants around a set of islands. In the controllable condition, the pilot flies to an island based on the color of the plane that the participant selected, allowing participants' choices to influence the subsequent state. In the uncontrollable condition the pilot flies in a particular route without considering the plane that the participant selected. (b) A schematic of the task is shown. The controllable and uncontrollable conditions of the task alternate covertly. After every six exploratory trials, participants are probed about the subsequent state ("Where is the plane most likely to fly next?") and the condition ("Which pilot was flying the plane?"). (c) On every exploratory trial, participants see the current island and two planes. They can choose one plane to see where it will fly. (d) Participants can use their knowledge of the transition structure during the exploratory trials to select choices that reveal which pilot is flying the plane. Only discriminatory choices can be used to disentangle the current condition.

In order to make learning more challenging, the transitions were probabilistic throughout the four runs of the task. Participants were told that sometimes air traffic control instructed the pilot where to fly, but that most of the time pilots followed the usual pattern. During the first and third runs, the planes transitioned as described above $90 \%$ of the time, and went to each of the other two islands (the current and other "off-rule" islands) $5 \%$ of the time. During the second and fourth runs, we changed the transition probabilities to make the task more difficult. The planes transitioned as described above $80 \%$ of the time, and to each of the other two islands $10 \%$ of the time. Shifts in these transition probabilities were not instructed. Whereas previous studies have manipulated controllability by changing the probability that an action will yield a specific reward outcome (Dorfman \& Gershman, 2019; Metcalfe et al., 2010), this task equates statetransition probabilities across the controllable and uncontrollable conditions, eliminating the potential confound of coupling controllability with predictability (Ligneul, 2021). The controllable and uncontrollable conditions alternated eleven times during the 360 exploratory trials of the task. Although the total number of exploratory trials remained the same for all participants, the number of exploratory trials prior to each shift in condition was variable (between 18 to 42 trials), and the order of these condition intervals was shuffled between participants (i.e., all participants experience the same numbers of exploratory trials prior to condition shifts, just in a different 
sequence). Participants were prompted to take a short break three times during the task. Following the break, they were reminded of the transition structures for both conditions.

\section{State Predictions}

After every six exploratory trials, participants were asked to predict where a particular plane would fly next. Participants were sequentially asked about the two different colored planes that could depart from a given island. In the uncontrollable condition, correct predictions required selecting the same island for both planes because the color of the plane (i.e., the choice the participant made) did not influence which island appeared next. Conversely, accurate responses in the controllable condition required making divergent predictions about where the different colored planes would fly because the next island was contingent upon the color of the plane. In this way, state predictions could reveal the participant's current beliefs about control. Feedback was randomly given on only one of the two state predictions to incentivize learning without revealing the underlying condition. For reinforced trials, correct predictions yielded an image of a chest full of treasure and incorrect responses led to an image of an empty treasure chest. On the half of trials that were unreinforced, a treasure chest was not displayed. In total, there were 60 prediction pairs (120 state predictions).

\section{Condition Predictions}

Following the state prediction pairs, participants were asked which pilot was flying the plane as a direct index of control beliefs that did not depend on accurate knowledge of the task structure. Participants saw a picture of the two pilots side-by-side and then selected the pilot they believed to have been flying the plane, reflecting their beliefs about the current condition. No feedback was given on any of these 60 trials.

\section{Training Phase}

Prior to the task, participants were given explicit instructions about where each pilot would fly and had practice playing the game. The training proceeded in the same manner as the task except that all state transitions for the exploratory trials were deterministic and participants were told which pilot was flying the plane. As in the task, participants were asked to predict where the plane would fly and which pilot was flying the plane. In the training, unlike during the task, feedback was given for every prediction. The practice consisted of 48 exploratory trials and eight sets of predictions (where a set of predictions consists of 2 state predictions and 1 condition prediction), split evenly between the controllable and uncontrollable condition.

\section{Post-task Questions}

After completing the game, participants answered six questions about the structure of the task. For each pilot, they were asked where they would fly next based on either the color of the plane (controllable condition only) or the current location (uncontrollable condition only). Participants were shown all three destinations and asked to select the correct one for each pilot.

\section{Secondary Measures}

As we were interested in the underlying role of working memory in the ability to assess control, participants completed a short list-sorting working memory task from the NIH Toolbox Cognition 
Battery, which has previously been demonstrated to have high reliability and good construct validity in child and adolescent samples (Tulsky et al., 2013). Participants were shown up to seven items from the same category (e.g., food or animals) displayed one at a time on an iPad. When the image was displayed, participants heard the name of the item. Participants were instructed to repeat the items back to the experimenter in order of increasing size. In the next part of the task, participants were presented with up to 7 items from two distinct categories and asked to sort all the items from one category by size prior to sorting the items from the other category by size. Thus, this task required maintaining and manipulating items in mind in order to perform well. In our analyses, except for the mediation analysis where we used raw scores, we included the age-corrected working memory scores to assess whether differences in working memory, independent of age, were related to behavioral performance. The working memory task was added to the experimental protocol after data collection had begun. Thus, data from one male and five female adolescents are not included in any of the working memory analyses, resulting in a sample size of 84 participants.

To ensure that age was not confounded with differences in age-normed reasoning ability, we also administered the Vocabulary and Matrix Reasoning subtests of the Wechsler Abbreviated Scale of Intelligence (WASI). We observed no significant age differences in age-normed WASI scores (Wechsler, 2011; see Supplementary Materials).

We also administered the internal locus of control questionnaire to assess subjective sense of control (Nowicki \& Strickland, 1973) and the MacArthur socioeconomic status questionnaire for exploratory analyses. Results are not reported here.

\section{Statistical Analyses}

To examine both linear and quadratic effects of age, we conducted likelihood ratio tests for logistic models and ANOVAs for linear models to determine whether the inclusion of age alone or age and age-squared as predictors in the model provided a significantly better fit. We report which model provided a better fit through model comparison, along with the corresponding statistics from the winning model. Continuous and interval predictors in the regression models were z-scored for interpretability. Age was included as a continuous variable, unless otherwise noted, and categorical age bins were applied for visualization. Age-squared was calculated by squaring the z-scored age. Behavioral analyses were performed using $R$ version 3.5.2 (R Core Team, 2017) and Matlab 2016a (Mathworks). All $p$-values reflect a two-tailed alpha threshold of $p<.05$. Mixed-effect models were conducted in $\mathrm{R}$ using the afex package Version .22-1 (Singmann et al., 2016). We used the optimizer "bobyqa" and set the number of model iterations to one million. The maximal model was specified to minimize Type I error (Barr et al., 2013), except where noted. If the maximal model did not converge or resulted in a singular fit, we reduced the random effects structure until the model converged. Details on model specification and full results can be found in the Supplementary Materials. Mediation analyses were performed using the mediation package in R (Tingley et al., 2014). Confidence intervals were estimated using 10,000 bootstrapped samples to test the significance of the mediation effects.

\section{Computational modeling}


To gain insight into the cognitive mechanisms underlying participants' choices and the source of potential age-related differences, we fit four models (the Spectator, Actor, Learned Transition Structure, and Task Set models) inspired by a previous study in adults using a more complex version of the task (Ligneul et al., 2020). These models formalize different ways of making predictions about the state that will be encountered next $\left(s^{\prime}\right)$ - by estimating transition probabilities based on states (Spectator model) or states and actions (Actor model); by estimating controllability based on the difference between the Spectator and Actor predictions (Learned Transition Structure model); or by inferring controllability based on perfect knowledge of the task transition structure (Task Set model), which affords the ability to make diagnostic interventions from the start of the task. The Task Set model is the only model that formalizes an inferential strategy and requires representation of the complete task structure in working memory. Thus, the set of models requires tracking an increasingly large set of transition probabilities, with inference requiring the maintenance and manipulation of these complex structures in working memory.

For all but the Task Set model, predictions for transitions that were experienced are updated using an error-driven process. The prediction error captures the difference between the experienced transition (which was coded as 1) and the predicted transition probability. The extent to which learned transition probabilities are updated by the most recent prediction error is governed by the learning rate $(\alpha)$. Predictions for the transitions that did not occur are decremented, ensuring that transition probabilities sum to 1 (see Supplementary Materials for details).

The Spectator model makes predictions about the subsequent state $\left(P\left(s^{\prime} \mid s\right)\right)$ based solely on states $(s)$, without taking actions into account, thus assuming environmental uncontrollability; Eq. 1).

$$
P\left(s^{\prime} \mid s\right) \leftarrow P\left(s^{\prime} \mid s\right)+\alpha_{s s^{\prime}}\left(1-P\left(s^{\prime} \mid s\right)\right)
$$

The Actor model makes these predictions $\left(P\left(s^{\prime} \mid s, a\right)\right)$ based on states $(s)$ and actions $(a)$, thus assuming environmental controllability; Eq. 2).

$$
P\left(s^{\prime} \mid s, a\right) \leftarrow P\left(s^{\prime} \mid s, a\right)+\alpha_{s a s^{\prime}}\left(1-P\left(s^{\prime} \mid s, a\right)\right)
$$

The remaining two models, the Learned Transition Structure model and the Task Set model, both dynamically estimate the causal influence of actions over state transitions by comparing predictions about subsequent transitions from the Spectator and Actor models $\left(P\left(s^{\prime} \mid s, a\right)-\right.$ $P\left(s^{\prime} \mid s\right)$ ). This expected difference, $\Omega$, represents an online estimate of the degree of controllability of the environment. In a controllable environment, actions contribute to predictions about the upcoming state and therefore, there will be an action for which $P\left(s^{\prime} \mid s, a\right)>P\left(s^{\prime} \mid s\right)$. Higher $\Omega$ values provide evidence that the environment is more controllable. Unlike the Spectator and Actor models, both of the controllability models have a second-order learning rate $\left(\alpha_{\Omega}\right)$, which governs the updating of the expected difference in the predictive capability of the Spectator versus the Actor model (i.e. $P\left(s^{\prime} \mid s, a\right)-P\left(s^{\prime} \mid s\right)$; Eq. 3$)$. 


$$
\Omega \leftarrow \Omega+\alpha_{\Omega}\left(P\left(s^{\prime} \mid s, a\right)-P\left(s^{\prime} \mid s\right)-\Omega\right)
$$

In order to transform $\Omega$ into a probability between 0 and 1 that can be used for prediction, and to capture distinct ways in which estimates of control may be biased, $\Omega$ is transformed into an "arbitrator", $\omega$, using a two-parameter sigmoidal function (Eq. 4).

$$
\omega=\frac{1}{1+\exp \left(-\beta_{\Omega}\left(\Omega-\operatorname{bias}_{\Omega}\right)\right)}
$$

The 'bias' parameter $\left(\right.$ bias $_{\Omega}$ ) in the sigmoidal function acts as a threshold above which $\Omega$ is interpreted as evidence in favor of a controllable environment, and thus can capture persistent biases toward estimates of controllability or uncontrollability. The slope parameter of the sigmoidal transformation $\left(\beta_{\Omega}\right)$ determines the extent to which the most likely first-order model (i.e. the Spectator or the Actor depending on whether $\Omega$ is above or below the 'bias' estimate) is given priority when making predictions about a future state.

For the Learned Transition Structure and Task Set models, state predictions are made by weighting the spectator and actor model using the arbitrator $\omega$ (Eq. 5).

$$
p\left(S^{\prime}=i\right)=\omega \max _{j=1: 3} p\left(S^{\prime}=i \mid S_{j}, A\right)+(1-\omega) p\left(S^{\prime}=i \mid S\right)
$$

The difference between the Learned Transition Structure model and the Task Set model is the manner through which the transition structure is learned. The Learned Transition Structure model updates the state-state and state-action-state transition probabilities from experience, whereas the Task Set model uses prior knowledge about the rules governing the task transition structure to infer the degree of controllability of the environment and make state predictions. Therefore, in the Task Set model, transition probabilities are fully pre-learned and set to either 1 or 0 based on the rules governing state-state and state-action-state transitions that were explicitly instructed during the training phase. As transition probabilities for this model are not updated, the first-order learning rate is set to 0 . Within the Task Set model, the Spectator and Actor models explicitly represent the two possible task sets that can alternate covertly during the experiment. The arbitrator, $\omega$, derived from $\Omega$, represents the arbitration between task sets when making a prediction. Thus, the Task Set model reflects the use of an inferential (or hypothesistesting) strategy, as opposed to the Learned Transition Structure model that reflects a continuous updating based on experience.

For all four models, the probability that the participant predicts the next state is determined by a softmax equation (Eq. 6). An inverse temperature parameter ( $\left.\beta_{\text {choice }}\right)$ controls choice consistency with respect to predicted transitions. Higher values for $\beta_{\text {choice }}$ implies that the participant systematically selected the most likely transition, whereas a value close to 0 implies that the participant randomly guessed on prediction trials. 


$$
p(\text { prediction }=i)=\frac{\exp \left(\beta_{\text {choice }} p\left(S^{\prime}=i\right)\right)}{\sum_{j=1}^{j=3} \exp \left(\beta_{\text {choice }} p\left(S^{\prime}=i\right)\right)}
$$

In total, the Learned Transition Structure model has five free parameters: two learning rates, two parameters that transform $\Omega$ into $\omega$, and an inverse temperature. The Task Set model has only four free parameters, as the first-order learning rate is set to 0 . The Spectator model and the Actor model both have two free parameters: a learning rate and an inverse temperature.

Model variables were updated in the same manner following every exploratory trial and on state prediction trials that ended with feedback. However, only state predictions were used to constrain model fits, as there were no correct choices during exploration (even though there were informative or uninformative choices). Bayesian Information Criterion (BIC) was used for model comparison. A full description of the model space, fitting, and model comparison procedures is available in the Supplementary Materials.

\section{Information-theoretic analyses}

To characterize age-related differences in the pattern of exploratory choices, we quantified how evenly the space of possible state-action pairs was explored throughout the task. To do so, mutual information was calculated a posteriori for each participant by computing the shared information between states and actions for all exploratory trials $I\left(S_{t}, A_{t}\right)$. Greater mutual information reflects greater diversity in action selection, whereas less mutual information reflects a tendency to repeatedly select the same action in a given state. We used the MIToolbox (https://github.com/Craigacp/MIToolbox) to compute mutual information.

\section{RESULTS}

\section{State prediction accuracy}

During the task, participants were probed about where a plane would fly next as an indirect way to assess their beliefs of environmental controllability. We first examined how the accuracy of these revealed assessments of control changed as a function of the true degree of environmental control over the course of the task, as well as how accuracy was modulated by individual differences in working memory, a hypothesized component process of controllability inferences. We fit a generalized mixed-effects logistic regression model to participants' choices on state prediction trials, collapsing across runs with $80 \%$ versus $90 \%$ transition probabilities between states (as there were no significant differences; see Supplementary Materials). We calculated the proportion of diagnostic choices on every set of six exploratory trials for each participant, reflecting the informativeness of interventions prior to assessing control. Age, condition, state prediction trial number, proportion of diagnostic exploratory choices, and agecorrected working memory were included as predictors in the model. Interactions were included in the model except between working memory and proportion of diagnostic exploratory choices, as we wanted to limit the size of the model and had no a priori hypotheses about these interactions. The addition of the quadratic age term did not provide a better fit than the model including linear age alone $\left(X^{2}(12)=9.77, p=.636\right)$. 
Participants across age were more accurate at making predictions in the controllable condition $\left(X^{2}(1)=12.71, p=.0004\right.$; Fig. 2a), although performance for both conditions was well above chance even in the youngest individuals. As we hypothesized, state prediction accuracy increased with age $\left(X^{2}(1)=32.88, p<.0001\right)$, suggesting that accuracy in assessments of controllability improved across development. Participants' accuracy improved over the course of the task $\left(X^{2}(1)=11.35, p=.0008\right)$, providing evidence of learning. There was also a significant effect of diagnostic choice on state prediction accuracy $\left(X^{2}(1)=7.3, p=.007\right)$, such that a greater number of diagnostic interventions led to more accurate controllability assessments. With age, individuals became better at using diagnostic interventions to assess environmental controllability (age-by-diagnostic choice interaction: $X^{2}(1)=7.82, p=.005$ ). In addition, diagnostic exploratory choices influenced prediction accuracy differently for the controllable and uncontrollable condition (condition-by-diagnostic choice interaction: $X^{2}(1)=5.07, p=.02$ ), with the degree of diagnostic choices promoting accurate state predictions to a greater extent in the uncontrollable condition. This result provides further evidence of participants' bias toward controllability beliefs, as it suggests that stronger evidence (provided by diagnostic exploratory choices) was needed to confirm that actions were ineffective than to confirm their causal efficacy. No other main effects or interactions reached significance ( $p$ 's $\geq .07$; see Supplementary Table S1 for a full table of results). We repeated this analysis in the full sample after removing working memory as a predictor, and the same pattern of significant and nonsignificant effects remained (see Supplementary Table S2).

To characterize the effect of reversals of controllability conditions, we examined whether accuracy and response times changed after these shifts in conditions. We performed separate mixed-effects logistic regressions, including trials since reversal, age, and condition as predictors of state or condition prediction accuracy in the models. We found a significant effect of trials since reversal on state predictions $\left(X^{2}(1)=4.02, p=.04\right)$, suggesting that performance is lowest following the reversal and improves with time (see Supplementary Materials for modeling details and full results for state and condition predictions; condition predictions did not show a similar effect). Next, we investigated whether response times differed by age, condition or as a function of prediction trials since reversal. We found that participants across age were faster to predict the next state in the controllable condition (see Supplementary Materials for analysis details and results for both state and condition predictions). As response times are typically slower when choice uncertainty is higher (Koriat \& Ackerman, 2010; Zakay \& Tuvia, 1998), these findings parallel the more accurate performance on state predictions when actions are causal. 

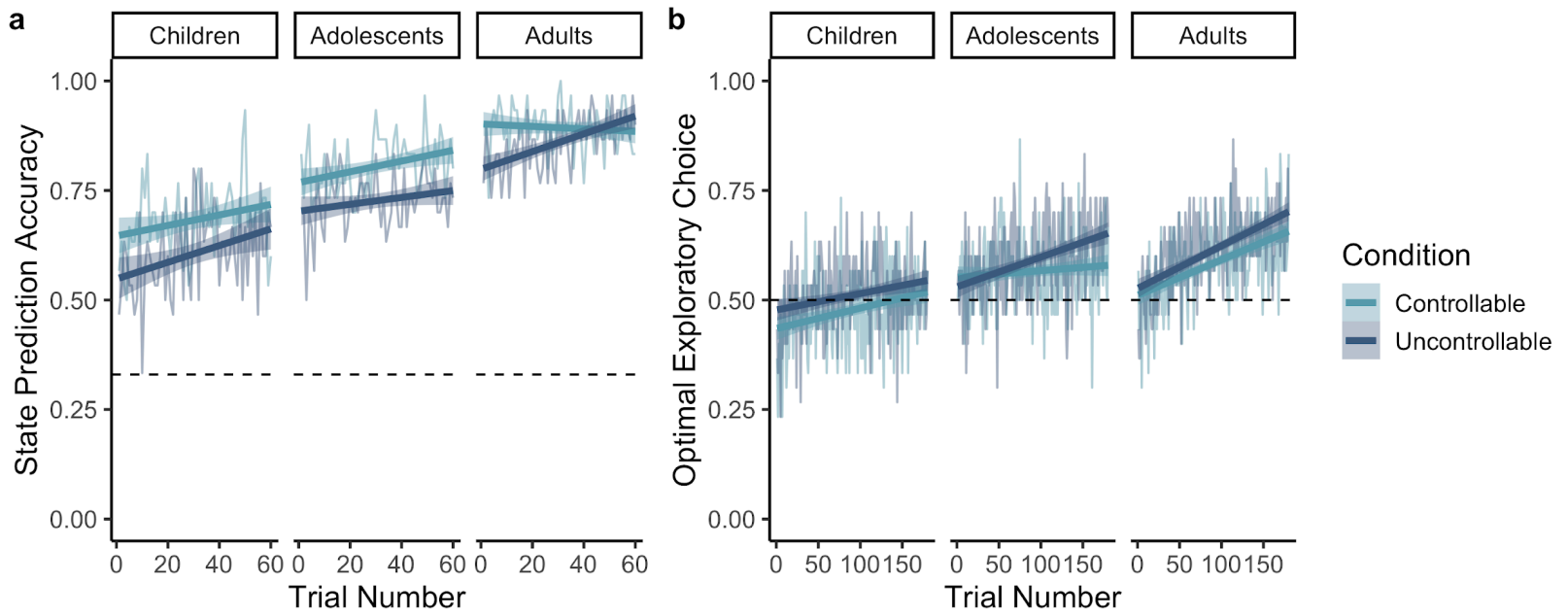

Figure 2. Performance on state predictions and exploratory trials over the course of the task. (a) Accuracy on state predictions and (b) diagnostic choices on exploratory trials are shown as a function of age group (children: 8-12 years, adolescents: 13-17 years, adults: 18-25 years), trial, and condition. Thinner lines represent the average performance for each group and condition, whereas the thicker lines represent the line of best fit with a $95 \%$ confidence interval. Chance performance is denoted by dashed lines.

\section{Condition prediction accuracy}

In addition to state predictions, which required learning the structure of the task, participants were also asked to make binary judgments about which pilot was flying the plane. These condition predictions acted as a coarser measure of control beliefs, as they did not directly rely on knowledge of the task transition structure. We repeated the mixed-effects logistic regression analysis to predict participants' choices on condition prediction trials, again collapsing across runs with $80 \%$ versus $90 \%$ transition probabilities between states (as there were no significant differences; see Supplementary Materials). Age, condition, condition prediction trial number, proportion of diagnostic exploratory choices, and age-corrected working memory were included as predictors in the model. As for the state prediction mixed-effects model, we did not allow working memory and proportion of diagnostic exploratory choices to interact. Including an agesquared term in the model did not improve the fit $\left(X^{2}(12)=4.26, p=.978\right)$. As with state predictions and in line with our hypothesis, explicit judgments about environmental controllability were more accurate in older individuals $\left(X^{2}(1)=26.95, p<.0001\right)$. Accuracy was higher in the controllable condition, which reflected a bias toward beliefs of control, and performance improved over the course of the task (condition: $X^{2}(1)=9.38, p=.002$; trial: $X^{2}(1)=4.86, p=$ .03). Selecting informative choices during the exploratory trials was associated with more accurate explicit judgments $\left(X^{2}(1)=17.32, p<.0001\right)$. As with state predictions, older individuals were significantly better at using diagnostic choices on the exploratory trials to predict the condition (age-by-diagnostic choice interaction: $X^{2}(1)=16.78, p<.0001$ ). Greater working memory performance was indicative of more accurate predictions about the condition $\left(X^{2}(1)=3.73, p=.05\right)$. None of the other main effects or interactions reached significance ( $p$ 's > 0.1 ; see Supplementary Table $S 3$ for full results). As with state predictions, we repeated this 
analysis without working memory to allow for a full sample size. All significant effects remained significant and nothing that was previously non-significant became significant (see Supplementary Table S4).

To better assess the specific role of working memory in controllability assessments, we asked whether age-related differences in working memory might account for developmental improvements in condition prediction accuracy. First, we confirmed that raw scores from the working memory task increased with age $(\beta=.33$, SE $=.1, t=3.11 p=.003)$ and related to greater accuracy on condition predictions while controlling for age $(\beta=.23$, SE $=.1, t=2.17 p=$ .033). Then we tested whether working memory mediated this relation between age and condition prediction accuracy. This analysis revealed that working memory partially mediated the relationship between age and condition prediction accuracy (standardized indirect effect: $.07,95 \%$ confidence interval: [.005 : .19], $p=.028$; standardized direct effect: $.34,95 \%$ confidence interval: [.12:.53], $p=.002$ ), highlighting working memory as a core cognitive mechanism supporting the detection of control. A similar pattern of results was observed for state predictions (see Supplementary Materials).

\section{Exploratory choices}

The age differences evident in participants' inferences of control suggest that individuals might be relying on different intervention strategies on exploratory trials. On every exploratory trial there were two choices, only one of which was diagnostic as to the condition. To investigate the informativeness of interventions during exploratory trials across age, we fit a generalized mixedeffects logistic regression with age, condition, trial, and all interaction terms as predictors. The model that included the addition of an age-squared term provided a better fit $\left(X^{2}(4)=10.26, p=\right.$ 0.036). Participants selected the diagnostic exploratory choice more in the uncontrollable than controllable condition $\left(X^{2}(1)=16.05, p<.0001\right.$; Fig. $\left.2 b\right)$. While we had no a priori predictions about such a difference, this effect may be consistent with the bias toward controllability beliefs revealed by participants' state predictions, and suggest that more evidence was required for participants to confirm that their actions were not causal. A significant effect of trial reflects that knowledge about the task structure may improve over the course of the task, facilitating more frequent diagnostic interventions in the later trials $\left(X^{2}(1)=7.97, p=.005\right)$. A significant age-bytrial interaction revealed that adults selected the diagnostic choice more frequently as the task progressed, whereas younger individuals showed less change in selecting the diagnostic choice over the course of the task $\left(X^{2}(1)=5.24, p=.02\right)$. No other effects reached significance (see Supplementary Table S5 for full results). Thus, adults may be better at learning to use knowledge of the task rules to make informative interventions.

The age-related improvement in learning to make diagnostic exploratory choices was paralleled by an age-related increase in the tendency to perform specific actions in specific states of the environment (e.g., repeatedly selecting the green plane when on the palm island). Mutual information, which captures the amount of information gained about actions by knowing about the states in which they were selected (and vice versa), increased with age (rho $=0.42, p<$ 
0.001), suggesting that younger individuals tended to evenly explore available actions within a state, while older individuals demonstrated more rigid coupling of states and actions. While adults may rely on an accurate mental representation of the task structure to make informative interventions that are diagnostic as to the current degree of controllability, younger individuals may thus aggregate over state-action-state observations to statistically estimate the degree of causality between their actions and outcomes. By exhibiting greater variability in their exploration of actions, younger individuals can generate a broader set of observations that may facilitate learning from experience, rather than through the use of the explicit task knowledge.

\section{Computational modeling of behavior}

Computational modeling allows us to formally articulate components of the cognitive processes that may contribute to an individual's choices. Here, we investigated how the computations used to assess environmental controllability changed from childhood to adulthood. We fit four computational models, described in detail in the Computational Modeling section of the Supplementary Materials, to each participant's data. These models differed in how they evaluated contingencies between states and actions and how they incorporated knowledge about the task structure that could be gleaned from the instructions and the training phase. The Spectator model and the Actor model assumed static beliefs of uncontrollability or controllability, respectively. The Learned Transition Structure model and Task Set model both incorporated dynamic beliefs about environmental controllability, but predictions about the transition structure were updated from experience in the Learned Transition Structure model and from explicit task instructions in the Task Set model.

At the group level, model comparison revealed a clear advantage for the Task Set model, indicating that the majority of participants made controllability inferences using a hypothesistesting approach in which the task rules (Fig. 3a) are represented explicitly (exceedance probability > 0.999; estimated frequency: $80.5 \%$; BIC difference with the second best model = 2516; Fig. 3b) (see Supplementary Materials for analysis of age differences in model parameter estimates). Model and parameter recoverability were high, ensuring interpretability of the computational modeling results (see Supplementary Materials). While the majority of children, adolescents, and adults were best fit by the Task Set model, children displayed the greatest strategy heterogeneity. Younger participants had a higher propensity than older individuals to rely on the Spectator model, which did not take into account the effects of one's actions in the prediction of upcoming states. Importantly, at the group level, all four models fit participants' choice behavior better than a random model (no learning, 0 free parameters): the overall BIC of the random model greatly exceeded the overall BIC of the worst-fitting model (i.e., the Spectator model; $\triangle \mathrm{BIC}$ : 3707). Examination of the relative advantage of the models within the comparison set, captured by the difference in their BIC values, provides evidence of systematic age-related changes in the information participants took into account when making controllability assessments. A positive correlation between age and the relative advantage of the Actor model over the Spectator model reflects that with age, individuals increasingly incorporated the contingencies between states and actions into their model of the task structure (rho $=0.39, p<$ 0.001; Supplementary Fig. S1a). Furthermore, a significant positive correlation between age and the relative advantage of the Task Set model over the Learned Transition Structure model 
suggests an increased reliance on explicit rule-based knowledge with age (rho $=0.48, p<$ 0.001; Supplementary Fig. S1b). Thus, individuals both considered the causal influence of their actions, and incorporated knowledge of the rule structure into their mental representation of the task to a greater extent with increasing age.

We next examined model-derived controllability estimates from the best-fitting Task Set model to validate their relation to participants' controllability assessments. In the model, participants' inferred controllability beliefs were reflected in the value of the arbitrator $(\omega)$. Whereas estimates of controllability for some participants reflected shifts in inferences of controllability that mirrored the true causal structure of the environment (Fig. 3c), others reflected a bias toward uncontrollability beliefs (Fig. 3d). As parameter estimates were derived by fitting the model to participants' state predictions, we used the condition predictions, which served as an entirely independent dataset, to assess how well binary choices about the current condition were predicted by the Task Set model. A logistic regression revealed that model-derived estimates of controllability, indexed by $\omega$, were strongly related to condition predictions (one-sample t-test on individual regression slopes: $t(89)=8.60, p<0.001$ ), and increasingly so with age (correlation between slopes and age: rho $=0.32, p=0.002$ ).

a

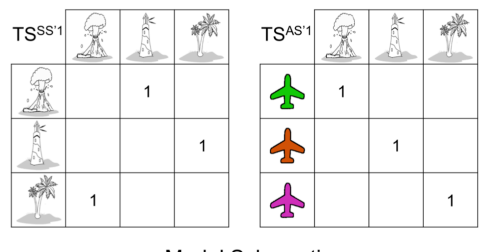

Model Schematic

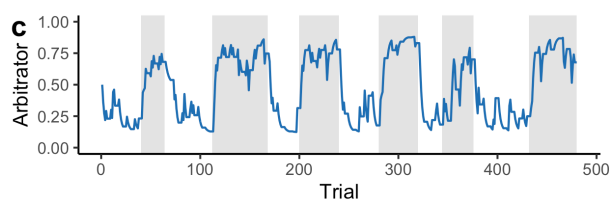

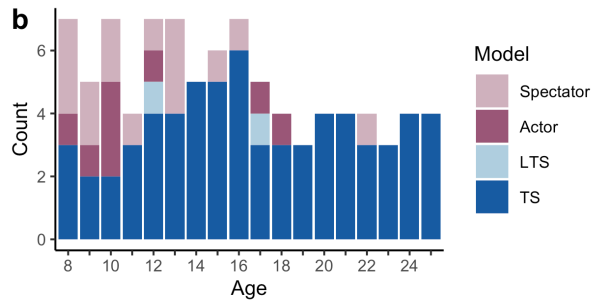

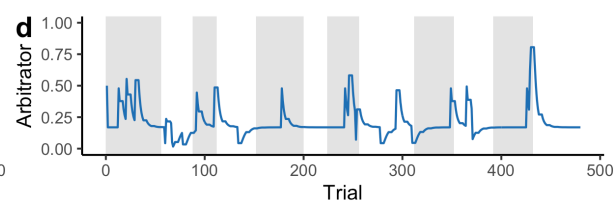

e
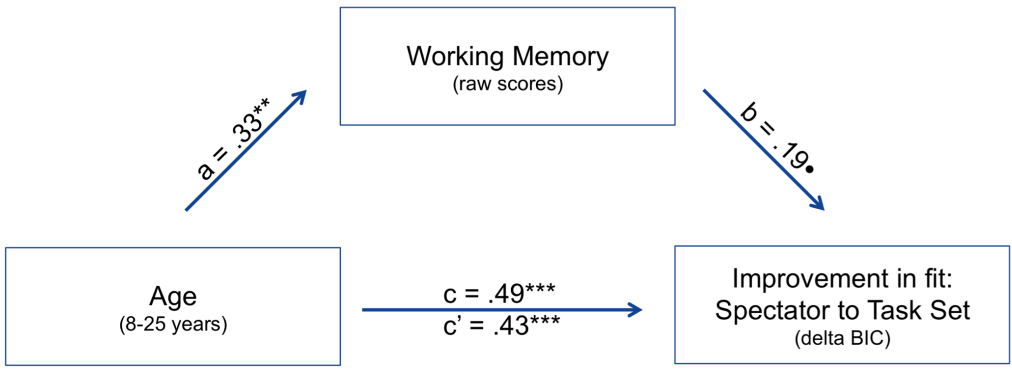

Figure 3. Model comparison and working memory mediation analysis for the best-fitting Task Set Model. (a) Task Set rules that govern how the state-state (SS'1) or state-action-state (SAS'1) models are updated after each trial. (b) The number of participants best fit by each model as a function of age. (c-d) Dynamics of the arbitrator are plotted for two example participants. Higher values reflect greater inferred controllability. Shaded areas reflect the controllable condition and white reflects the uncontrollable condition. (e) Working memory partially mediated the relation between age and improvement in quality of fit between the Spectator and Task Set model. Path a shows the regression 
coefficient for age-related improvements in working memory. Path b shows the regression coefficient for the influence of working memory on the improvement in model fit between the Spectator and Task Set models, while controlling for age. Path c' and c show regression coefficients for the influence of age on improvement in fit ( $\triangle B I C)$ with and without including working memory, respectively. The following abbreviations are used: LTS: Learned Transition Structure model; TS: Task Set model. ${ }^{* *}$ Denotes $p<.01 ;{ }^{* \star *}$ denotes $p<.001 ; \bullet p=.068$.

As working memory mediated the age-related improvement in assessment of controllability, we next tested whether working memory related to individuals' use of the simpler Spectator model, which requires holding in mind only the transitions between states, versus the other, more complex models that additionally take into account the consequences of actions, and thus require tracking a greater number of state transitions. We hypothesized that individuals with better working memory might show greater use of these more complex mental models of their environment, which would be reflected by a larger difference in quality of fit $(\triangle \mathrm{BIC})$ between the simpler and more complex models. Indeed, the difference in model fit between the Spectator and Task Set models increased with age $(\beta=.49$, SE $=.1, t=5.12 p<.001)$. A formal mediation analysis revealed working memory as a significant partial mediator between age and $\triangle \mathrm{BIC}$ for the Spectator and Task Set models (standardized indirect effect: .06, 95\% confidence interval: [.004: .16], $p=.028$; standardized direct effect: .43, 95\% confidence interval: [.24 : .62 ], $p<.001$; Fig. 3e). Working memory also showed a significant partial mediation of the relation between age and $\triangle \mathrm{BIC}$ between the Spectator model and all other models that incorporate the consequences of actions (i.e., the Learned Transition Structure and Actor models; see Supplementary Materials). This suggests that working memory contributes to agerelated increases in the use of mental models that incorporate actions, and their consequences, into assessments of control.

Whereas older individuals used task structure knowledge to make more informative interventions on exploratory trials, younger individuals exhibited greater randomness in their exploratory choices. To investigate whether the random exploration evident in children constitutes a resource-rational way of generating evidence for outcome prediction when inferential abilities are not as robust, we simulated data for each of the four models, varying whether interventions were entirely diagnostic or random. The simulations show that consistently selecting the diagnostic exploratory choice only facilitated controllability assessment for the Task Set model (Fig. 4a). For the other models that do not implement inferential strategies, random interventions yielded better performance. Empirical data matched these qualitative trends, such that individuals best fit by the Task Set model who made more diagnostic interventions (as determined by a median-split on the proportion of diagnostic choices across all participants) exhibited greater accuracy on condition predictions (Fig. 4b). In contrast, individuals best fit by any of the other three non-inferential models showed better performance if they explored more randomly. Thus, random exploration may provide younger learners with a compensatory mechanism for predicting outcomes when working memory capacity is not yet sufficient to represent and make inferences based on the full task transition structure. 
We reasoned that if age-related improvement in controllability assessment stemmed from the synergistic development of diagnostic interventions and inferential processes, then this improvement should be more pronounced in individuals who selected the diagnostic exploratory choice more frequently. Accordingly, we found that condition prediction accuracy increased with age in the half of participants (median-split) who more frequently made diagnostic interventions $(71+/-14 \%$ of diagnostic choices, $r=0.61, p<.001)$. In contrast, for the half of participants who explored more randomly or frequently selected the uninformative choice $(40+/-16 \%$ of diagnostic choices), there was no age-related improvement in condition predictions $(r=0.18, p$ $=0.25$, difference in correlation coefficients: $z=2.43, p=0.008$, Fig. $4 \mathrm{c}$ ). This pattern of findings was qualitatively similar for state prediction accuracy (see Supplementary Materials and Supplementary Fig. S4).
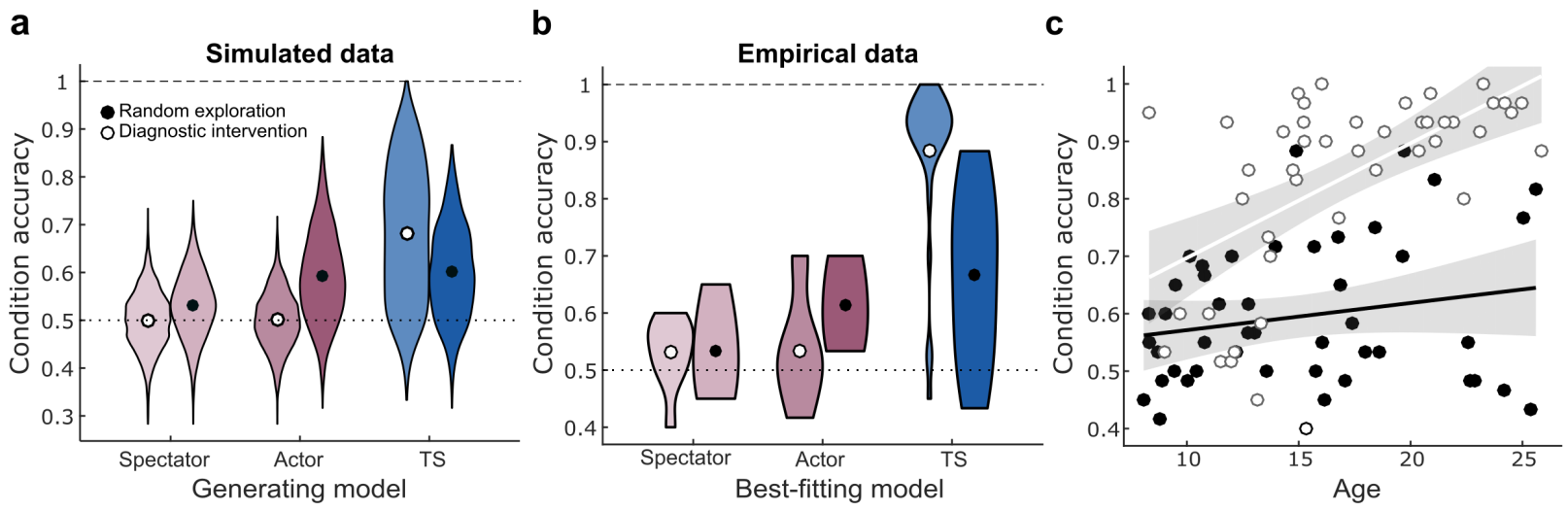

Figure 4. Simulated and empirical data for condition prediction accuracy as a function of diagnostic interventions. (a) Simulations reveal that consistently selecting the diagnostic exploratory choice is only beneficial for the Task Set (TS) model. Random exploration yields better controllability assessments for all other models. (b) A median-split of participants based on their proportion of diagnostic interventions closely matches model predictions and shows a unique performance benefit of diagnostic choices for individuals best fit by the Task Set model. Given that only two participants were best fit by the Learned Transition Structure model, it was not included in these analyses. (c) A median-split analysis revealed that age-related improvements in condition prediction accuracy were driven largely by participants who made more diagnostic choices (open circles) whereas no significant age-related improvement was evident for participants who explored more randomly (filled circles). Shaded areas represent $95 \%$ confidence intervals. Abbreviations: TS: Task Set.

\section{Explicit Knowledge}

Our model comparison results suggest that many participants use the task rules to ascertain the current condition by making diagnostic choices and that this may become the dominant strategy as individuals grow older. Given that such explicit knowledge about the task transition structure might account for age-related improvements in accuracy, following the game we probed participants about their explicit knowledge of the rules by which the two pilots flew. As perfect knowledge of the transition structure was quite common ( $82 \%$ of participants), we performed a chi-squared test to assess age differences in perfect explicit knowledge of the task transition structure between children, adolescents, and adults. This analysis revealed a significant difference in the distribution of participants who accurately reported all six transitions $\left(X^{2}(2)=\right.$ $6.537, p=0.038)$. Additional pairwise chi-squared tests confirmed that the proportion of children $\left(X^{2}(1)=6.405, p=0.011\right)$ and adolescents $\left(X^{2}(1)=5.192, p=0.023\right)$ who accurately answered all six questions significantly differed from adults, revealing that a greater proportion of adults 
had perfect knowledge of the task structure. However, no difference was observed between the proportion of children and adolescents who responded correctly to all the questions $\left(X^{2}(1)=\right.$ $.089, p=0.766)$. Thus, explicit knowledge of the task rules is more prevalent in adults than younger individuals, supporting the increased use of a hypothesis-testing strategy with age.

\section{DISCUSSION}

In this study, we investigated developmental changes in the ability to detect the controllability of the environment and select informative interventions that reveal its causal structure. Children, adolescents, and adults completed a task that covertly alternated between a controllable and uncontrollable condition whose causal structure could be discovered through exploratory interventions. Throughout the task, participants were frequently probed about their beliefs of environmental controllability, allowing us to study controllability detection with high temporal resolution. Children tended to make more random exploratory choices, whereas older participants made more interventions that provided diagnostic evidence of the current condition. We found that children distinguished between controllable and uncontrollable conditions with a high degree of accuracy, however accuracy of these assessments improved with age into adulthood. Across all ages, controllability assessments were more accurate in the controllable versus the uncontrollable condition. Computational modeling showed that age-related improvements in controllability assessment stemmed from an increased ability to represent the structure of contingencies between states and actions and to use that knowledge to infer the current state of control by making informative interventions. Working memory contributed to this developmental transition by supporting older individuals' use of more complex mental representations of the task structure. In contrast, younger individuals' greater reliance on random exploration may be a resource-rational strategy for learning environmental contingencies when working memory abilities are still developing (Lieder \& Griffiths, 2020; Ruel et al., 2020). Taken together, our findings suggest that a qualitative shift toward greater use of inferential reasoning may lead to more accurate controllability assessments with age, which may facilitate better detection of opportunities to engage in goal-directed action across development.

Consistent with an extensive literature suggesting the biological and psychological importance of environmental control (Leotti et al., 2010; Maier \& Seligman, 1976; Mineka \& Hendersen, 1985), we found that participants of all ages were more prone to infer control rather than the lack thereof, suggesting a bias toward beliefs of controllability. Such a bias may indeed be beneficial. Beliefs about controllability are proposed to generalize to novel situations where the environmental structure is unknown (Huys \& Dayan, 2009; Maier \& Seligman, 2016; Moscarello \& Hartley, 2017). Generalizing controllability beliefs can promote active exploratory behavior and cognitive engagement, which facilitate the discovery of action-outcome affordances in novel environments (Huys \& Dayan, 2009; Moscarello \& Hartley, 2017). In contrast, as famously demonstrated in early studies of learned helplessness (Maier \& Seligman, 1976), experiences of uncontrollability decrease exploration, attention (Minor et al., 1984), and contingency learning in novel contexts (Overmier \& Seligman, 1967), perhaps reflecting reduced engagement of goaldirected learning processes when past experience has suggested that they are likely to be 
ineffective. Such generalization effects have been proposed to reflect an adaptive calibration of cognitive effort and behavioral strategies to the degree of agency afforded by an environment (Moscarello \& Hartley, 2017), a process that can occur over both local and developmental timescales (Frankenhuis et al., 2013; Kubala et al., 2012). Such a bias toward controllability beliefs early in life (van Elk et al., 2015), may be particularly advantageous as it reduces the potential opportunity costs of curtailing exploration and goal-directed learning when uncertainty about the true degree of environmental controllability is still high.

Whereas adults appeared to use a mental model of the task structure to promote informative interventions and accurate causal inferences, children and adolescents showed more difficulty using instructed information to guide their exploratory choices and controllability assessments. At the start of the task, participants were taught the action-outcome contingencies that applied in the controllable condition and the sequence of transitions that occurred in the uncontrollable condition. Prior work suggests that adults can rapidly learn to adjust their behavior according to instructed tasks rules (Cole et al., 2013). In contrast, younger individuals, who may not yet be adept at using mental representations of a task's structure to inform choices (Cohen et al., 2020; Decker et al., 2016; Potter et al., 2017), tend to favor learning from experience over the use of instructed information (Brace et al., 2006; Decker et al., 2015). Children's near-chance selection of the diagnostic exploratory choice, as well as their broad exploration of the potential actions available in each state, is consistent with a vast literature highlighting a shift from greater reliance on exploration to exploitation with age (Gopnik, 2020; Gopnik et al., 2015; Lucas et al., 2014; Meder et al., 2020; Nussenbaum \& Hartley, 2019; Wilson et al., 2021), and may reflect a greater propensity of younger individuals to estimate controllability from the statistics of experienced outcomes, rather than using task structure knowledge to perform diagnostic hypothesis tests. Children's greater reliance on random exploration has previously been shown to benefit learning (Sumner et al., 2019) and may reflect a resource-rational strategy in younger individuals whose working memory abilities are not as robust (Lieder \& Griffiths, 2020; Ruel et al., 2020). An open question is whether the observed age differences in the accurate assessment of controllability and explicit knowledge about the task structure might be mitigated in a context where the statistical regularities between states and actions must be discovered through experience, in the absence of explicit instruction.

Under multiple theoretical accounts, estimation of environmental controllability acts as a metalevel learning process that can be used to adapt one's cognition and behavior to situational demands. Controllability assessments allow for the appropriate assignment of credit to oneself or to external causes when learning the value of actions (Dorfman et al., 2019; Ligneul et al., 2020) and amplifies learning signals for agentic choices (Cockburn et al., 2014). Like the ability to accurately detect the state of environmental controllability assessed here, such use of controllability inferences to inform reinforcement learning has been found to increase with age (Cohen et al., 2020). The rational allocation of cognitive control to support goal-directed behavior is also proposed to depend on assessments of environmental controllability (Boureau et al., 2015; Dorfman \& Gershman, 2019; Frömer et al., 2021; Shenhav et al., 2013). In line with theoretical proposals that deliberative action selection should be preferentially engaged in controllable environments (Huys \& Dayan, 2009; Moscarello \& Hartley, 2017), adults can use 
dynamic estimates of controllability to arbitrate between using learned action-outcome contingencies to achieve goals versus simpler reflexive strategies that assume no contingency between actions and outcomes (Csifcsák et al., 2019; Dorfman \& Gershman, 2019). Our present findings that controllability assessments improve into young adulthood suggest that children may similarly be less adept at adjusting their reward-motivated behavior according to the degree of environmental controllability. However, future work is necessary to better understand whether arbitration between these value-based learning systems across controllable and uncontrollable contexts changes with age.

The ability to determine when one's actions are consequential provides foundational knowledge about the structure of the environment that organizes an individual's learning and decision making. The present study demonstrated that from childhood to adulthood, individuals were able to detect dynamic changes in environmental controllability, and that the accuracy of these assessments improved with age. Across development, we also found that assessments were biased toward beliefs of control, a tendency with potentially beneficial consequences for learning and mental health. Children, adolescents and adults exhibited a synergistic coupling between their exploratory behaviors and the nature of their learning strategies, which was dependent upon the cognitive resources available at their respective developmental stages. Collectively, these findings reveal a qualitative shift across development in the underlying strategies through which individuals recognize when actions can be leveraged for their benefit.

\section{Data \& Code Availability}

Data and code will be made available on Open Science Framework upon publication.

\section{Acknowledgements}

We are grateful to the families who participated in this study. We thank Katerina Frangulova and Sophia Mascialino for their assistance with data collection. This work was supported by a Jacobs Foundation Early Career Research Fellowship (to C.A.H.), a Klingenstein-Simons Fellowship in Neuroscience (to C.A.H.), a National Science Foundation CAREER grant 1654393 (to C.A.H.), a Fyssen Foundation postdoctoral award (to R.L.), a National Science Foundation Graduate Research Fellowship DGE1839302 (to H.A.R.), the NYU Vulnerable Brain Project, and the NIH-funded R90DA043849 NYU Training Program in Computational Neuroscience (to C.F.).

\section{Author Information}

H.A.R., R.L., \& C.A.H. designed the study. H.A.R. and C.F. collected the data. H.A.R., R.L., and C.F. performed analyses. H.A.R., R.L., \& C.A.H. drafted the manuscript. All authors provided feedback.

\section{Additional Information}

The authors declare no competing interests. 


\section{References}

Amso, D., \& Davidow, J. (2012). The development of implicit learning from infancy to adulthood: Item frequencies, relations, and cognitive flexibility. Developmental Psychobiology, 54(6), 664-673. https://doi.org/10.1002/dev.20587

Amso, D., Haas, S., McShane, L., \& Badre, D. (2014). Working memory updating and the development of rule-guided behavior. Cognition, 133(1), 201-210. https://doi.org/10.1016/j.cognition.2014.06.012

Barnett, L., Barrett, A. B., \& Seth, A. K. (2009). Granger Causality and Transfer Entropy Are Equivalent for Gaussian Variables. Physical Review Letters, 103(23), 238701. https://doi.org/10.1103/PhysRevLett.103.238701

Barr, D. J., Levy, R., Scheepers, C., \& Tily, H. J. (2013). Random effects structure for confirmatory hypothesis testing: Keep it maximal. Journal of Memory and Language, 68(3). https://doi.org/10.1016/j.jml.2012.11.001

Boureau, Y.-L., Sokol-Hessner, P., \& Daw, N. D. (2015). Deciding How To Decide: Self-Control and Meta-Decision Making. Trends in Cognitive Sciences, 19(11), 700-710. https://doi.org/10.1016/j.tics.2015.08.013

Brace, J. J., Morton, J. B., \& Munakata, Y. (2006). When Actions Speak Louder Than Words: Improving Children's Flexibility in a Card-Sorting Task. Psychological Science, 17(8), 665-669. https://doi.org/10.1111/j.1467-9280.2006.01763.x

Chambon, V., Théro, H., Vidal, M., Vandendriessche, H., Haggard, P., \& Palminteri, S. (2020). Information about action outcomes differentially affects learning from self-determined versus imposed choices. Nature Human Behaviour, 1-13. https://doi.org/10.1038/s41562-020-0919-5

Cockburn, J., Collins, A. G. E., \& Frank, M. J. (2014). A Reinforcement Learning Mechanism Responsible for the Valuation of Free Choice. Neuron, 83(3), 551-557. 
https://doi.org/10.1016/j.neuron.2014.06.035

Cohen, A. O., Nussenbaum, K., Dorfman, H. M., Gershman, S. J., \& Hartley, C. A. (2020). The rational use of causal inference to guide reinforcement learning strengthens with age. Npj Science of Learning, 5(1), 1-9. https://doi.org/10.1038/s41539-020-00075-3

Cole, M. W., Laurent, P., \& Stocco, A. (2013). Rapid instructed task learning: A new window into the human brain's unique capacity for flexible cognitive control. Cognitive, Affective \& Behavioral Neuroscience, 13(1), 1-22. https://doi.org/10.3758/s13415-012-0125-7

Cook, C., Goodman, N. D., \& Schulz, L. E. (2011). Where science starts: Spontaneous experiments in preschoolers' exploratory play. Cognition, 120(3), 341-349. https://doi.org/10.1016/j.cognition.2011.03.003

Crone, E. A., Wendelken, C., Donohue, S., van Leijenhorst, L., \& Bunge, S. A. (2006). Neurocognitive development of the ability to manipulate information in working memory. Proceedings of the National Academy of Sciences of the United States of America, 103(24), 9315-9320. https://doi.org/10.1073/pnas.0510088103

Csifcsák, G., Melsæter, E., \& Mittner, M. (2019). Intermittent Absence of Control during Reinforcement Learning Interferes with Pavlovian Bias in Action Selection. Journal of Cognitive Neuroscience, 1-18. https://doi.org/10.1162/jocn_a_01515

Decker, J. H., Lourenco, F. S., Doll, B. B., \& Hartley, C. A. (2015). Experiential reward learning outweighs instruction prior to adulthood. Cognitive, Affective, \& Behavioral Neuroscience, 15(2), 310-320. https://doi.org/10.3758/s13415-014-0332-5

Decker, J. H., Otto, A. R., Daw, N. D., \& Hartley, C. A. (2016). From Creatures of Habit to GoalDirected Learners Tracking the Developmental Emergence of Model-Based Reinforcement Learning. Psychological Science, 27(6), 848-858. https://doi.org/10.1177/0956797616639301

Dorfman, H. M., Bhui, R., Hughes, B. L., \& Gershman, S. J. (2019). Causal Inference About Good and Bad Outcomes. Psychological Science, 30(4), 516-525. 
https://doi.org/10.1177/0956797619828724

Dorfman, H. M., \& Gershman, S. J. (2019). Controllability governs the balance between Pavlovian and instrumental action selection. Nature Communications, 10(1), 1-8. https://doi.org/10.1038/s41467-019-13737-7

Frankenhuis, W. E., Gergely, G., \& Watson, J. S. (2013). Infants May Use Contingency Analysis to Estimate Environmental States: An Evolutionary, Life-History Perspective. Child Development Perspectives, 7(2), 115-120. https://doi.org/10.1111/cdep.12024

Frömer, R., Lin, H., Dean Wolf, C. K., Inzlicht, M., \& Shenhav, A. (2021). Expectations of reward and efficacy guide cognitive control allocation. Nature Communications, 12(1), 1030. https://doi.org/10.1038/s41467-021-21315-z

Goldstein, M. H., King, A., \& West, M. (2003). Social interaction shapes babbling: Testing parallels between birdsong and speech. Proceedings of the National Academy of Sciences of the United States of America, 100(13), 8030-8035. https://doi.org/10.1073/pnas.1332441100

Gopnik, A. (2020). Childhood as a solution to explore-exploit tensions. Philosophical Transactions of the Royal Society B: Biological Sciences, 375(1803), 20190502. https://doi.org/10.1098/rstb.2019.0502

Gopnik, A., Glymour, C., Sobel, D. M., Schulz, L. E., Kushnir, T., \& Danks, D. (2004). A theory of causal learning in children: Causal maps and Bayes nets. Psychological Review, 111(1), 3-32. https://doi.org/10.1037/0033-295X.111.1.3

Gopnik, A., Griffiths, T. L., \& Lucas, C. G. (2015). When younger learners can be better (or at least more open-minded) than older ones. Current Directions in Psychological Science, 24(2), 87-92.

Gunnar, M. R. (1980). Contingent Stimulation: A Review of its Role in Early Development. In S. Levine \& H. Ursin (Eds.), Coping and Health (pp. 101-119). Springer US. https://doi.org/10.1007/978-1-4684-1042-6_6 
Haggard, P. (2017). Sense of agency in the human brain. Nature Reviews Neuroscience, 18(4), 196-207. https://doi.org/10.1038/nrn.2017.14

Hansen, D. M., \& Jessop, N. (2017). A context for self-determination and agency: Adolescent developmental theories. In Development of self-determination through the life-course (pp. 27-46). Springer Science + Business Media. https://doi.org/10.1007/978-94-0241042-6_3

Heckhausen, J. (2011). Agency and control striving across the life span. Handbook of Life-Span Development, 183-212.

Huys, Q. J. M., \& Dayan, P. (2009). A Bayesian formulation of behavioral control. Cognition, 113(3), 314-328. https://doi.org/10.1016/j.cognition.2009.01.008

Katzman, P. L., \& Hartley, C. A. (2020). The value of choice facilitates subsequent memory across development. Cognition, 199, 104239. https://doi.org/10.1016/j.cognition.2020.104239

Kelso, J. A. S. (2016). On the Self-Organizing Origins of Agency. Trends in Cognitive Sciences, 20(7), 490-499. https://doi.org/10.1016/j.tics.2016.04.004

Klyubin, A. S., Polani, D., \& Nehaniv, C. L. (2005). Empowerment: A universal agent-centric measure of control. 2005 IEEE Congress on Evolutionary Computation, 1, 128-135. https://doi.org/10.1109/CEC.2005.1554676

Koriat, A., \& Ackerman, R. (2010). Choice latency as a cue for children's subjective confidence in the correctness of their answers. Developmental Science, 13(3), 441-453. https://doi.org/10.1111/j.1467-7687.2009.00907.x

Kubala, K. H., Christianson, J. P., Kaufman, R. D., Watkins, L. R., \& Maier, S. F. (2012). Shortand Long-Term Consequences of Stressor Controllability in Adolescent Rats. Behavioural Brain Research, 234(2), 278-284. https://doi.org/10.1016/j.bbr.2012.06.027

Kuhn, D., Garcia-Mila, M., Zohar, A., Andersen, C., White, S. H., Klahr, D., \& Carver, S. M. (1995). Strategies of Knowledge Acquisition. Monographs of the Society for Research in 
Child Development, 60(4), i-157. JSTOR. https://doi.org/10.2307/1166059

Kushnir, T., \& Gopnik, A. (2005). Young Children Infer Causal Strength From Probabilities and Interventions. Psychological Science, 16(9), 678-683. https://doi.org/10.1111/j.14679280.2005.01595.x

Lagnado, D. A., \& Sloman, S. (2004). The advantage of timely intervention. Journal of Experimental Psychology. Learning, Memory, and Cognition, 30(4), 856-876. https://doi.org/10.1037/0278-7393.30.4.856

Leotti, L. A., lyengar, S. S., \& Ochsner, K. N. (2010). Born to Choose: The Origins and Value of the Need for Control. Trends in Cognitive Sciences, 14(10), 457-463. https://doi.org/10.1016/j.tics.2010.08.001

Lieder, F., \& Griffiths, T. L. (2020). Resource-rational analysis: Understanding human cognition as the optimal use of limited computational resources. Behavioral and Brain Sciences, 43. https://doi.org/10.1017/S0140525X1900061X

Ligneul, R. (2021). Prediction or Causation? Towards a Redefinition of Task Controllability. Trends in Cognitive Sciences. https://doi.org/10.1016/j.tics.2021.02.009

Ligneul, R., Mainen, Z., Ly, V., \& Cools, R. (2020). Stress-sensitive inference of task controllability. BioRxiv, 2020.11.19.390393. https://doi.org/10.1101/2020.11.19.390393

Lucas, C. G., Bridgers, S., Griffiths, T. L., \& Gopnik, A. (2014). When children are better (or at least more open-minded) learners than adults: Developmental differences in learning the forms of causal relationships. Cognition, 131(2), 284-299.

https://doi.org/10.1016/j.cognition.2013.12.010

Ly, V., Wang, K. S., Bhanji, J., \& Delgado, M. R. (2019). A Reward-Based Framework of Perceived Control. Frontiers in Neuroscience, 13. https://doi.org/10.3389/fnins.2019.00065

Maier, S. F., \& Seligman, M. E. P. (1976). Learned Helplessness: Theory and Evidence. Journal of Experimental Psychology: General, 105, 3-46. https://doi.org/10.1037/0096- 
3445.105.1.3

Maier, S. F., \& Seligman, M. E. P. (2016). Learned helplessness at fifty: Insights from neuroscience. Psychological Review, 123(4), 349-367. https://doi.org/10.1037/rev0000033

Meder, B., Wu, C. M., Schulz, E., \& Ruggeri, A. (2020). Development of directed and random exploration in children [Preprint]. PsyArXiv. https://doi.org/10.31234/osf.io/7zhxv

Mesman, J., van IJzendoorn, M. H., \& Bakermans-Kranenburg, M. J. (2009). The many faces of the Still-Face Paradigm: A review and meta-analysis. Developmental Review, 29(2), 120-162. https://doi.org/10.1016/j.dr.2009.02.001

Metcalfe, J., Eich, T. S., \& Castel, A. D. (2010). Metacognition of agency across the lifespan. Cognition, 116(2), 267-282. https://doi.org/10.1016/j.cognition.2010.05.009

Meulemans, T., Van der Linden, M., \& Perruchet, P. (1998). Implicit sequence learning in children. Journal of Experimental Child Psychology, 69(3), 199-221. https://doi.org/10.1006/jecp.1998.2442

Mineka, S., \& Hendersen, R. W. (1985). Controllability and predictability in acquired motivation. Annual Review of Psychology, 36(1), 495-529.

Minor, T. R., Jackson, R. L., \& Maier, S. F. (1984). Effects of task-irrelevant cues and reinforcement delay on choice-escape learning following inescapable shock: Evidence for a deficit in selective attention. Journal of Experimental Psychology: Animal Behavior Processes, 10(4), 543-556. https://doi.org/10.1037/0097-7403.10.4.543

Moscarello, J. M., \& Hartley, C. A. (2017). Agency and the Calibration of Motivated Behavior. Trends in Cognitive Sciences, 21(10), 725-735. https://doi.org/10.1016/j.tics.2017.06.008

Nadel, J., Carchon, I., Kervella, C., Marcelli, D., \& Réserbat Plantey, D. (1999). Expectancies for social contingency in 2-month-olds. Developmental Science, 2(2), 164-173. https://doi.org/10.1111/1467-7687.00065 
Nowicki, S., \& Strickland, B. R. (1973). A locus of control scale for children. Journal of Consulting and Clinical Psychology, 40(1), 148-154. https://doi.org/10.1037/h0033978

Nussenbaum, K., Cohen, A. O., Davis, Z. J., Halpern, D. J., Gureckis, T. M., \& Hartley, C. A. (2020). Causal Information-Seeking Strategies Change Across Childhood and Adolescence. Cognitive Science, 44(9), e12888. https://doi.org/10.1111/cogs.12888

Nussenbaum, K., \& Hartley, C. A. (2019). Reinforcement learning across development: What insights can we draw from a decade of research? Developmental Cognitive Neuroscience, 40, 100733. https://doi.org/10.1016/j.dcn.2019.100733

Otto, A. R., Gershman, S. J., Markman, A. B., \& Daw, N. D. (2013). The curse of planning: Dissecting multiple reinforcement-learning systems by taxing the central executive. Psychological Science, 24(5), 751-761. https://doi.org/10.1177/0956797612463080

Overmier, J. B., \& Seligman, M. E. (1967). Effects of inescapable shock upon subsequent escape and avoidance responding. Journal of Comparative and Physiological Psychology, 63(1), 28-33. https://doi.org/10.1037/h0024166

Palminteri, S., Kilford, E. J., Coricelli, G., \& Blakemore, S.-J. (2016). The Computational Development of Reinforcement Learning during Adolescence. PLoS Computational Biology, 12(6). https://doi.org/10.1371/journal.pcbi.1004953

Pearl, J. (2009). Causality (2nd ed.). Cambridge University Press.

Potter, T. C. S., Bryce, N. V., \& Hartley, C. A. (2017). Cognitive components underpinning the development of model-based learning. Developmental Cognitive Neuroscience, 25, 272280. https://doi.org/10.1016/j.den.2016.10.005

R Core Team. (2017). R: A language and environment for statistical computing. (3.4.0) [Computer Software]. R Foundation for Statistical Computing. http://www.R-project.org/ Raab, H. A., \& Hartley, C. A. (2018). The Development of Goal-Directed Decision-Making. In R. Morris, A. Bornstein, \& A. Shenhav (Eds.), Goal-Directed Decision Making (pp. 279_ 308). Academic Press. https://doi.org/10.1016/B978-0-12-812098-9.00013-9 
Rieber, M. (1969). Hypothesis testing in children as a function of age. Developmental Psychology, 1(4), 389-395. https://doi.org/10.1037/h0027697

Ruel, A., Devine, S., \& Eppinger, B. (2020). Resource-rational approach to meta-control problems across the lifespan. PsyArXiv. https://doi.org/10.31234/osf.io/s5ard

Ruggeri, A., Markant, D. B., Gureckis, T. M., Bretzke, M., \& Xu, F. (2019). Memory enhancements from active control of learning emerge across development. Cognition, 186, 82-94. https://doi.org/10.1016/j.cognition.2019.01.010

Saffran, J. R., Aslin, R. N., \& Newport, E. L. (1996). Statistical learning by 8-month-old infants. Science (New York, N.Y.), 274(5294), 1926-1928.

Salge, C., Glackin, C., \& Polani, D. (2014). Empowerment-An Introduction. In M. Prokopenko (Ed.), Guided Self-Organization: Inception (pp. 67-114). Springer. https://doi.org/10.1007/978-3-642-53734-9_4

Shenhav, A., Botvinick, M. M., \& Cohen, J. D. (2013). The Expected Value of Control: An Integrative Theory of Anterior Cingulate Cortex Function. Neuron, 79(2), 217-240. https://doi.org/10.1016/j.neuron.2013.07.007

Singmann, H., Bolker, B., Westfall, J., Aust, F., Højsgaard, S., Fox, J., \& Mertens, U. (2016). Afex: Analysis of Factorial Experiments. Comprehensive R Archive Network (CRAN) [R]. https://github.com/singmann/afex

Sloman, S. A., \& Lagnado, D. A. (2005). Do We "do"? Cognitive Science, 29(1), 5-39. https://doi.org/10.1207/s15516709cog2901_2

Sobel, D. M., \& Kushnir, T. (2006). The importance of decision making in causal learning from interventions. Memory \& Cognition, 34(2), 411-419. https://doi.org/10.3758/BF03193418

Sobel, D. M., \& Legare, C. H. (2014). Causal learning in children. Wiley Interdisciplinary Reviews. Cognitive Science, 5(4), 413-427. https://doi.org/10.1002/wcs.1291

Sobel, D. M., \& Sommerville, J. A. (2010). The Importance of Discovery in Children's Causal Learning from Interventions. Frontiers in Psychology, 1. 
https://doi.org/10.3389/fpsyg.2010.00176

Steyvers, M., Tenenbaum, J. B., Wagenmakers, E.-J., \& Blum, B. (2003). Inferring causal networks from observations and interventions. Cognitive Science, 27(3), 453-489. https://doi.org/10.1207/s15516709cog2703_6

Sumner, E., Li, A. X., Perfors, A., Hayes, B., Navarro, D., \& Sarnecka, B. W. (2019). The Exploration Advantage: Children's instinct to explore allows them to find information that adults miss. PsyArXiv. https://doi.org/10.31234/osf.io/h437v

Takahashi, D. Y., Liao, D. A., \& Ghazanfar, A. A. (2017). Vocal Learning via Social Reinforcement by Infant Marmoset Monkeys. Current Biology, 27(12), 1844-1852. https://doi.org/10.1016/j.cub.2017.05.004

Tingley, D., Yamamoto, T., Hirose, K., Keele, L., \& Imai, K. (2014). mediation: R Package for Causal Mediation Analysis. Journal of Statistical Software, 59(5). https://doi.org/10.18637/jss.v059.i05

Tulsky, D. S., Carlozzi, N. E., Chevalier, N., Espy, K. A., Beaumont, J. L., \& Mungas, D. (2013). V. Nih Toolbox Cognition Battery (cb): Measuring Working Memory. Monographs of the Society for Research in Child Development, 78(4), 70-87. https://doi.org/10.1111/mono.12035

van Elk, M., Rutjens, B. T., \& van der Pligt, J. (2015). The development of the illusion of control and sense of agency in 7- to-12-year old children and adults. Cognition, 145, 1-12. https://doi.org/10.1016/j.cognition.2015.08.004

Watson, J. S., \& Ramey, C. T. (1972). Reactions to response-contingent stimulation in early infancy. Merrill-Palmer Quarterly, 18(3), 219-227.

Wechsler, D. (2011). Wechsler Abbreviated Scale of Intelligence-Second Edition (WASI-II). NCS Pearson.

Wilson, R. C., Bonawitz, E., Costa, V. D., \& Ebitz, R. B. (2021). Balancing exploration and exploitation with information and randomization. Current Opinion in Behavioral Sciences, 
38, 49-56. https://doi.org/10.1016/j.cobeha.2020.10.001

Zakay, D., \& Tuvia, R. (1998). Choice latency times as determinants of post-decisional confidence. Acta Psychologica, 98(1), 103-115. https://doi.org/10.1016/S00016918(97)00037-1 